05

\title{
Нелинейное расщепление линии магнитоупругого резонанса в сильно возбужденном феррите
}

\author{
(C) В.С. Власов, ${ }^{1}$ В.Г. Шавров, ${ }^{2}$ В.И. Щеглов ${ }^{2}$ \\ ${ }^{1}$ Сыктывкарский государственный университет им. Питирима Сорокина, \\ 167001 Сыктывкар, Россия \\ ${ }^{2}$ Институт радиотехники и электроники им. В.А. Котельникова РАН, \\ 125009 Москва, Россия \\ e-mail: vshcheg@cplire.ru
}

Поступило в Редакцию 1 июня 2021 r.

В окончательной редакции 31 августа 2021 г.

Принято к публикации 1 сентября 2021 г.

Рассмотрено нелинейное расщепление линии магнитоупругого резонанса в сильно возбужденном феррите. Показано, что амплитуда возникающего при этом дополнительного резонанса имеет тот же порядок величины, что и амплитуда основного, а частота определяется верхней границей нелинейной частотной характеристики магнитной системы. Отмечен пороговый характер возникновения дополнительного резонанса. Установлена возможность управления частотой такого упругого резонанса путем изменения уровня возбуждающего магнитного поля, имеющая прикладное значение.

Ключевые слова: ферромагнитный резонанс, магнитоупругое взаимодействие, нелинейные колебания.

DOI: 10.21883/JTF.2022.01.51853.167-21

\section{Введение}

Мощные ультразвуковые колебания, возбуждаемые магнитострикционными преобразователями, находят широкое применение в различных областях техники $[1,2]$. Особый интерес представляет возбуждение гиперзвуковых колебаний на частотах до $10 \mathrm{GHz}$ и более. Весьма перспективным здесь является использование магнитоакустического резонанса [3-5]. Пленки ферритовгранатов открывают дополнительные возможности значительного расширения частотного диапазона преобразователей за счет возбуждения до нескольких сотен упругих мод по толщине структуры пленка-подложка [6]. Мощность возбуждения гиперзвука в таких преобразователях ограничивается на уровне десятков милливатт, что связано с параметрическим возбуждением обменных спиновых волн, по достижении определенного порога вызывающим сильное поглощение поступающего сигнала [7-9]. Предотвращение такого возбуждения достигается применением в качестве рабочего тела преобразователя нормально намагниченного тонкого ферритового диска. В такой геометрии частота ферромагнитного резонанса приходится на дно спектра обменных волн, в результате чего их возбуждение исключается [10-12]. В работах [13-16], реализующих такие условия, показана возможность увеличения уровня возбуждения гиперзвука на два-три порядка и более.

Однако в этих работах недостаточно внимания уделяется характеру амплитудночастотных свойств нелинейного резонанса магнитной системы, а также диссипации упругой системы, что не позволяет реализовать преимущества высокого уровня возбуждения в достаточной степени.
Настоящая работа посвящена более подробному рассмотрению резонансных частотных свойств обеих систем с целью выявить дополнительные возможности генерации мощного гиперзвука.

\section{1. Геометрия задачи и основные уравнения}

Общая геометрия задачи показана на рис. 1 [13-16]. Ее основу составляет ферритовая пластина толщиной $d$, намагниченная по нормали к ее плоскости.

Полагаем, что феррит по магнитным свойствам изотропен, а по упругим и магнитоупругим - обладает кубической симметрией, причем плоскость грани куба соответствует плоскости ферритовой пластины. Плоскость $O x y$ декартовой системы координат $O x y z$ совпадает с плоскостью пластины. Внешнее поле имеет вид: $\mathbf{H}=\left\{h_{x} ; h_{t} ; H_{0}\right\}$, где $H_{0}-$ постоянное подмагничивающее поле, $h_{x, y}$ - компоненты переменного поля. При возбуждении сдвиговых упругих колебаний вдоль оси $O z$ (т.е. при $u_{z}=0$, а также $\partial / \partial(x, y) \rightarrow 0$ ) упругие свойства среды описываются константой $c_{44}$, а магнитоупругие - константой $B_{2}$.

Уравнение движения для компоненты намагниченности $m_{x}$ имеет вид

$$
\begin{aligned}
& \frac{\partial m_{x}}{\partial t}=-\frac{\gamma}{1+\alpha^{2}}\left[\left(m_{y}+\alpha m_{x} m_{z}\right) H_{e z}\right. \\
& \left.-\left(m_{z}-\alpha m_{y} m_{z}\right) H_{e y}-\alpha\left(m_{y}^{2}+m_{z}^{2}\right) H_{e x}\right],
\end{aligned}
$$

а уравнения для $m_{y}$ и $m_{z}$ получаются из (1) циклической перестановкой $x, y, Z$. Здесь $\mathbf{m}=\mathbf{M} / M_{0}$ - нормирован- 


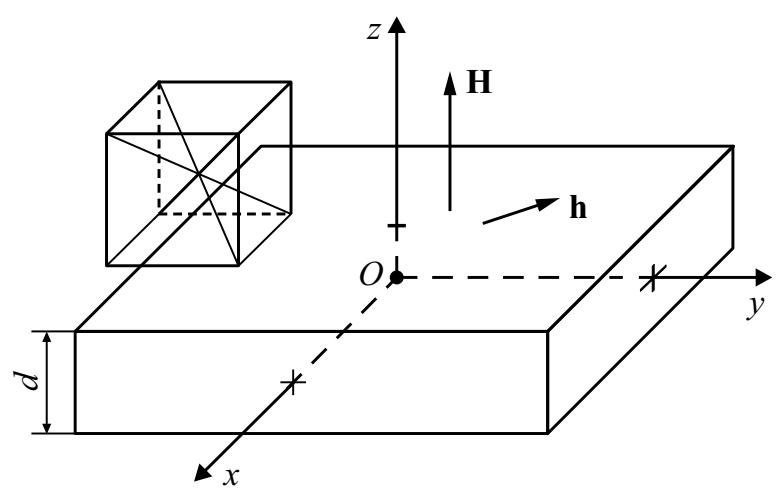

Рис. 1. Геометрия задачи.

ный вектор намагниченности, $M_{0}-$ намагниченность насыщения, $\alpha-$ константа затухания Гильберта.

Входящие в эти уравнения эффективные поля имеют вид

$$
\begin{gathered}
H_{e x}=h_{x}+H_{a x}, \\
H_{e y}=h_{y}+H_{a y}, \\
H_{e z}=H_{0}-4 \pi M_{0} m_{z}+H_{a z},
\end{gathered}
$$

где

$$
H_{a x}=-\frac{B_{2}}{M_{0}} m_{z} \frac{\partial u_{x}}{\partial z},
$$

где $u_{x}$ - компонента упругого смещения, а $H_{a y}$ и $H_{a z}$ получаются из (5) циклической перестановкой $x, y, z$.

Уравнение для компоненты смещения $u_{x}$ имеет вид

$$
\frac{\partial^{2} u_{x}}{\partial t^{2}}+2 \beta \frac{\partial u_{x}}{\partial t}-\frac{c_{44}}{\rho} \frac{\partial^{2} u_{x}}{\partial z^{2}}=0,
$$

а уравнение для $u_{y}$ получается из (6) заменой $x$ на $y$.

Граничные условия для компоненты смещения $u_{x}$ при свободных поверхностях пластины имеют вид

$$
\left.c_{44} \frac{\partial u_{x}}{\partial z}\right|_{z= \pm d / 2}=-B_{2} m_{x} m_{z},
$$

а условия для $u_{y}$ получаются из (7) заменой $x$ на $y$.

В рамках настоящей задачи переменное поле будем считать синусоидальным с правой круговой поляризацией:

$$
\begin{gathered}
h_{x}=h_{0} \sin (2 \pi f t), \\
h_{y}=-h_{0} \cos (2 \pi f t),
\end{gathered}
$$

где $h_{0}-$ амплитуда возбуждения.

Решение системы уравнений (1)-(6) с граничными условиями (7) методом Рунге-Кутты, подобное выполненное в работах [13-16], позволяет найти развитие во времени и частотные характеристики возбуждаемых переменным полем магнитных и упругих колебаний.

При расчете в настоящей работе использовались параметры материала, типичные для монокристалла ЖИГ (железо-иттриевого граната): $4 \pi M_{0}=1750 \mathrm{Gs}$,
$B_{2}(\mathrm{YIG})=6.96 \cdot 10^{5} \mathrm{~J} / \mathrm{m}^{-3}, \quad c_{44}=7.64 \cdot 10^{10} \mathrm{~J} / \mathrm{m}^{-3}$. Параметр затухания магнитной подсистемы (Гильберта) равнялся $\alpha=0.02$. Для упругой подсистемы использовались значения параметра затухания $\beta$ в интервале от $10^{6}$ до $10^{9} \mathrm{~s}^{-1}$, а также в качестве контрольного использовалось значение $\beta=0 \mathrm{~s}^{-1}$. Остальные параметры задачи выбирались такими, чтобы в отсутствие магнитоупругой связи при линейных колебаниях резонансные частоты однородной прецессии и первой моды упругих колебаний совпадали и равнялись $2800 \mathrm{MHz}$. При этом постоянное поле равнялось $H_{0}=2750 \mathrm{Oe}$, а толщина магнитной пластины $-0.06865 \cdot 10^{-6} \mathrm{~m}$. Амплитуда переменного поля для линейного режима составляла $h_{0}=0.01 \mathrm{Oe}$, для нелинейного $-10 \mathrm{Oe}$.

\section{2. Частотные характеристики нелинейных магнитоупругих колебаний}

В соответствии с постановкой задачи возбуждение колебаний в полной магнитоупругой системе осуществляется путем воздействия переменного поля на магнитную подсистему, поэтому сначала рассмотрим, каким образом в этом случае сказывается присутствие упругой подсистемы.

Обратимся к рис. 2, где показаны частотные характеристики амплитуды магнитных $(a)$ и упругих $(b)$ колебаний при различных уровнях связи между магнитной и упругой системами. Поскольку связь обусловлена магнитоупругим взаимодействием, то рассмотрим характеристики при двух различных значениях константы магнитоупругой связи - соответствующим ЖИГ (кривые 1 ) и в пять раз меньшим (кривые 2). При этом в первом случае связь является типичной для экспериментальной ситуации, а во втором - почти отсутствующей, но все же достаточной для возбуждения колебаний в упругой системе.

Сначала обратимся к случаю слабой связи (кривые 2). Здесь на рис. 2, $a$ наблюдается типичная характеристика нелинейного резонанса - треугольника, скошенного в сторону высоких частот за счет расстроечного механизма с максимумом около $3.02 \mathrm{GHz}$. Из рис. $2, b$ видно, что в этом случае частотная характеристика упругих колебаний имеет симметричный вид с центральным максимумом на резонансной частоте собственных упругих колебаний пластины $(2.8 \mathrm{GHz})$. При этом высокочастотный пик магнитного резонанса (кривая 2 на рис. $2, a$ ) на упругих колебаниях (кривая 2 на рис. $2, b$ ) практически не сказывается. На магнитной характеристике в области упругого резонанса $(2.8 \mathrm{GHz})$ хотя и наблюдается зубец, но весьма незначительный.

В случае сильной связи (кривые 1) обе характеристики существенно изменяются. На магнитной характеристике (кривая 1 на рис. $2, a$ ) в области упругого резонанса $(2.8 \mathrm{GHz})$ наблюдается резкий скачок сначала вверх, затем вниз, а сама характеристика в области от 2.94 до $3.02 \mathrm{GHz}$ поднимается вверх более чем на $20 \%$. 

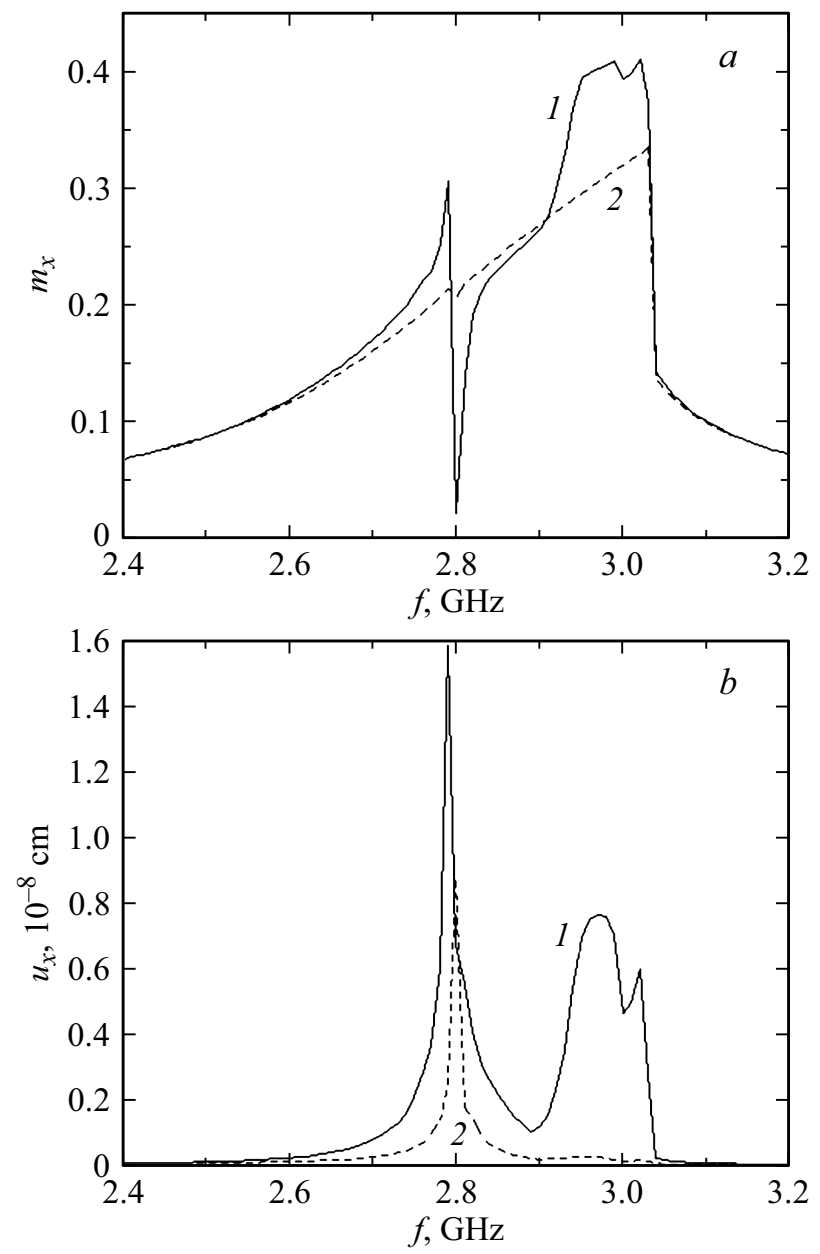

Рис. 2. Частотные характеристики амплитуды магнитных $(a)$ и упругих $(b)$ колебаний при различных значениях константы магнитоупругого взаимодействия: $1-B_{2}=B_{2}(\mathrm{YIG})$, $2-B_{2}=0.2 B_{2}(\mathrm{YIG})$.

На упругой характеристике основной пик значительно (около двух раз) усиливается и слегка смещается вниз (с 2.89 на $2.78 \mathrm{GHz}$ ), а в области от 2.94 до $3.02 \mathrm{GHz}$ возникает сильный дополнительный резонансный подъем, по амплитуде достигающий почти $50 \%$ от основного. Этот подъем имеет вид „отщепления“ от основного пика, что как раз соответствует подъему магнитной характеристики, т.е. находится в области действия расстроечного механизма. Заметим, что этот подъем не является цельным, а на его высокочастотной части имеется сравнительно небольшой второй дополнительный подъем, частота которого соответствует высокочастотному срезу магнитной характеристики $(3.02 \mathrm{GHz})$.

\section{3. Влияние уровня возбуждения на параметры упругого резонанса}

Рассмотрим зависимости частоты и амплитуды первого и второго дополнительных резонансов от уровня возбуждения, для чего обратимся к рис. 3 и 4.
На рис. 3 показаны зависимости частоты основного 1 и дополнительных первого 2 и второго 3 подъемов частотной характеристики амплитуды упругого смещения от амплитуды возбуждения.

Из рисунка видно, что основной подъем, соответствующий частоте парциального резонанса упругой системы $2.8 \mathrm{GHz}$ (кривая 1), существует при любом уровне возбуждения. Его частота от амплитуды возбуждения практически не зависит.

Первый дополнительный резонанс (кривая 2) существует только при уровне возбуждения выше 4 Ое вплоть до любого более высокого уровня. Частота этого резонанса зависит от амплитуды возбуждения линейно с угловым коэффициентом $0.0124 \mathrm{GHz} / \mathrm{Oe}$. Второй дополнительный резонанс (кривая 3 ) существует только в интервале возбуждения от 9 до 32 Ое. Его частота зависит от амплитуды возбуждения также линейно с тем же

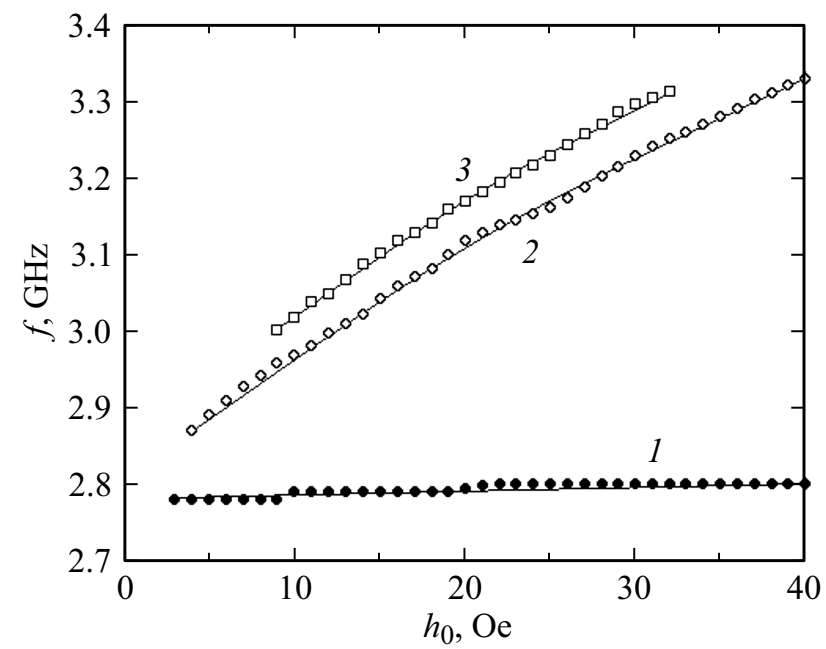

Рис. 3. Зависимости частоты основного (1) и дополнительных первого (2) и второго (3) резонансов частотной характеристики упругого смещения от амплитуды возбуждения.

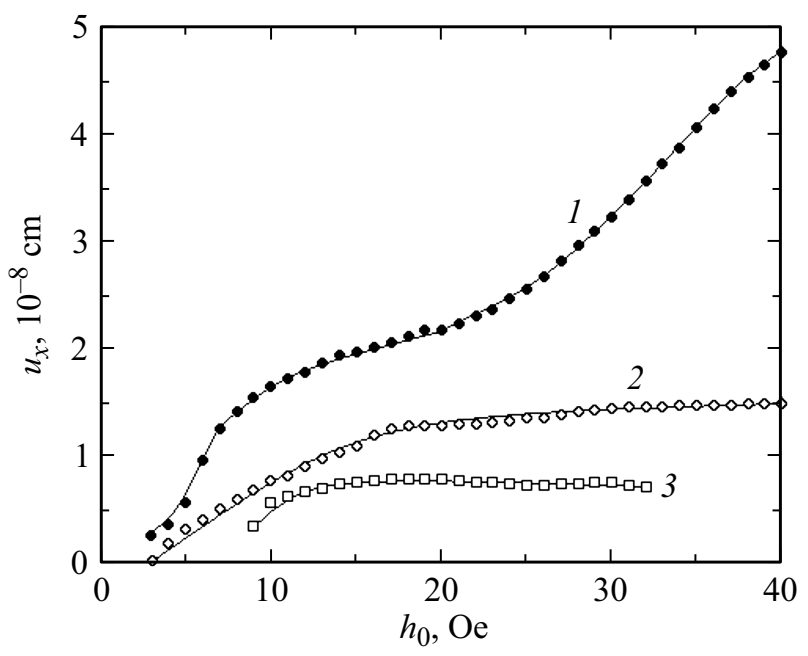

Рис. 4. Зависимости амплитуды основного (1) и дополнительных первого (2) и второго (3) резонансов частотной характеристики упругого смещения от амплитуды возбуждения. 
угловым коэффициентом, однако лежит выше частоты первого дополнительного резонанса на $0.06 \mathrm{GHz}$.

На рис. 4 приведены зависимости амплитуды основного и дополнительных первого и второго резонансов частотной характеристики упругого смещения от амплитуды возбуждения.

Из рисунка видно, что основной подъем, соответствующий парциальному резонансу упругой системы, т. е. частоте $2.8 \mathrm{GHz}$ (кривая 1 ), по мере роста уровня возбуждения также увеличивается. Сначала при амплитуде возбуждения менее 8 Ое амплитуда этого подъема возрастает довольно быстро, однако далее вплоть до уровня возбуждения около 25 Ое этот рост замедляется, что связано с частичной перекачкой энергии системы из основного в дополнительные резонансы. Начиная с уровня возбуждения $25 \mathrm{Oe}$, рост амплитуды снова ускоряется, видимо, в связи с ограничением перекачки энергии в дополнительные резонансы. При этом амплитуда первого дополнительного резонанса (кривая 2) сначала тоже растет, однако, начиная с уровня около $17 \mathrm{Oe}$, насыщается, после чего остается почти постоянной вплоть до высоких значений возбуждения. Амплитуда первого дополнительного резонанса в интервале возбуждения от 3 до 25 Ое, т.е. до ярко выраженного насыщения, составляет примерно в два раза меньше амплитуды основного резонанса в том же диапазоне. Амплитуда второго дополнительного резонанса (кривая 3) также сначала резко растет, но вскоре ее рост насыщается на уровне примерно в два раза ниже амплитуды первого дополнительного резонанса. При уровне возбуждения выше 32 Ое второй дополнительный резонанс сливается с первым, не имея собственной ярко выраженной вершины.

Приведенные в настоящем разделе результаты можно рассматривать как демонстрацию возможности перестройки частоты упругого резонанса в интервале от 2.86 до $3.32 \mathrm{GHz}$, т. е. в пределах 15\% при изменении уровня возбуждения от 5 до 40 Ое. Можно полагать, что такая возможность управления частотой упругого резонанса имеет определенное прикладное значение. Важным достоинством такого управления является отсутствие необходимости изменения механических параметров системы, в частности, толщины ферритовой пластины.

\section{4. Влияние уровня упругой диссипации}

Приведенные в предыдущем разделе результаты получены при специально выбранном достаточно низком уровне упругой диссипации. Такой выбор был сделан с целью наибольшей наглядности. Теперь рассмотрим, как проявляются описанные выше явления в широком интервале упругого затухания.

На рис. 5 показаны зависимости амплитуды основного и обоих дополнительных резонансов частотной характеристики упругого смещения от параметра упругой диссипации.

Из рисунка видно, что по мере увеличения диссипации амплитуды всех резонансов плавно спадают. При этом

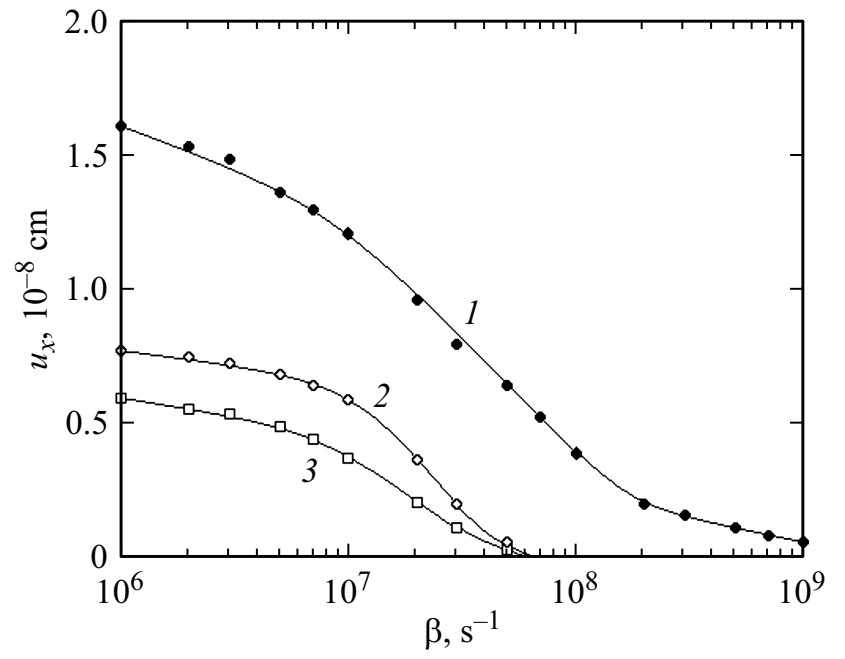

Рис. 5. Зависимости амплитуды основного (1) и дополнительных первого (2) и второго (3) резонансов частотной характеристики упругого смещения от параметра упругой диссипации. По горизонтальной оси принят логарифмический масштаб.

основной подъем, соответствующий парциальной частоте упругого резонанса пластины (кривая 1), остается достаточно ярко выраженным во всем принятом интервале изменения параметра $\beta$ от $10^{6}$ до $10^{9} \mathrm{~s}^{-1}$, только его амплитуда падает примерно в 20 раз. При этом резонансный подъем по частоте хотя и расширяется, но еще сохраняет свой индивидуальный характер, т.е. имеет форму центрального максимума со спадами в обе стороны. При дальнейшем увеличении затухания (за пределами рисунка) резонанс приобретает апериодический характер, т. е. повторяет скошенный в сторону высоких частот треугольный вид магнитного резонанса с уменьшающейся амплитудой.

Амплитуды обоих дополнительных резонансов (кривые 2 и 3) по мере увеличения затухания также падают, однако, начиная с уровня $\beta$ около $5 \cdot 10^{7} \mathrm{~s}^{-1}$, боковые резонансы сливаются с основным. Таким образом, четкое выделение как первого, так и второго дополнительных резонансов имеет место только тогда, когда параметр затухания составляет менее $5 \cdot 10^{7} \mathrm{~s}^{-1}$. Уменьшение параметра магнитного затухания $\alpha$ в 4 раза (от 0.020 до 0.005 ), хотя и приводит к слиянию обоих дополнительных резонансов друг с другом, но заметного изменения критического значения $\beta=5 \cdot 10^{7} \mathrm{~s}^{-1}$, при котором дополнительные резонансы сливаются с основным, не происходит.

Контрольная проверка показывает, что при уменьшении параметра затухания $\beta$ ниже $10^{6} \mathrm{~s}^{-1}$ вплоть до нуля (за левым краем рисунка) амплитуда всех резонансов ограничивается, основного - на уровне $1.7 \cdot 10^{-8} \mathrm{~cm}$, первого дополнительного - на уровне $0.8 \cdot 10^{-8} \mathrm{~cm} \mathrm{и}$ второго - на уровне $0.6 \cdot 10^{-8} \mathrm{~cm}$.

Таким образом, необходимыми условием осуществления управляемого уровнем возбуждения упругого резонанса является достаточно малая величина упругой 
диссипации, в конкретном случае менее $5 \cdot 10^{7} \mathrm{~s}^{-1}$, что для ЖИГ является вполне достижимым $[3,4]$.

\section{5. Развитие колебаний во времени}

Поскольку в сильно нелинейном режиме имеет место расщепление упругого резонанса, представляет интерес рассмотреть развитие колебаний во времени.

Обратимся к рис. 6, где показаны соответствующие развертки колебаний, происходящих после включения магнитного возбуждения. Рассмотрение выполнено на двух частотах, первая из которых соответствует основной частоте упругого резонанса $(2.80 \mathrm{GHz})$, а вторая центральной частоте главного дополнительного резонанса упругих колебаний $(2.97 \mathrm{GHz})$.

Из рис. 6, $a$ и $c$ можно видеть, что сразу после включения возбуждения амплитуда магнитных колебаний (рис. 6, a) резко увеличивается, так как в этот момент энергия возбуждения еще не успевает дойти до упру-
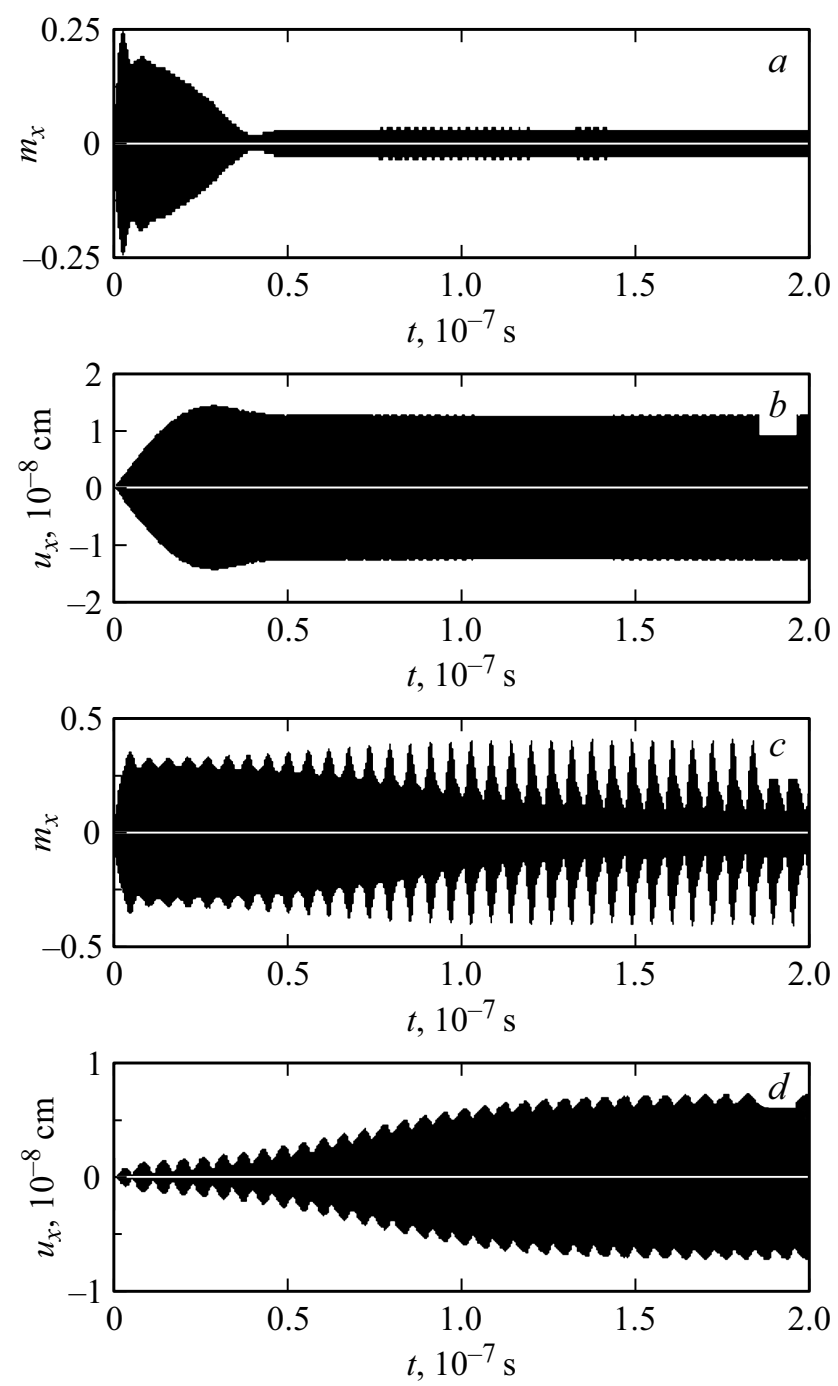

Рис. 6. Развитие во времени магнитных $(a, c)$ и упругих $(b, d)$ колебаний на разных частотах: $a, b-2.80 \mathrm{GHz}$ (основной резонанс); $c, d-2.97 \mathrm{GHz}$ (первый дополнительный резонанс). гой системы, амплитуда колебаний которой (рис. $6, b$ ) нарастает значительно медленнее. Далее амплитуда магнитных колебания постепенно уменьшается, а упругих увеличивается, что соответствует перекачке энергии из магнитной системы в упругую. Со времени около $0.5 \cdot 10^{-7}$ s наступает баланс энергий между системами, так что амплитуды колебаний в обеих системах устанавливаются на постоянном уровне. Такая картина соответствует обычному возбуждению колебаний в системе из двух связанных осцилляторов и имеет подобный вид в линейном режиме.

Не так, однако, обстоит дело на частоте дополнительного резонанса упругой системы. Как это видно из рис. $6, c$, магнитные колебания здесь также сначала резко увеличиваются, однако возбуждение упругих колебаний (рис. $6, d$ ), т. е. перекачка энергии из магнитной системы в упругую происходит здесь значительно медленнее, чем в предыдущем случае. В результате стационарный режим тех и других колебаний устанавливается только при времени около $1.5 \cdot 10^{-7} \mathrm{~s}$, т.е. в три раза большем, чем на частоте основного резонанса. Можно полагать, что столь сильное запаздывание возбуждения упругих колебаний обусловлено удалением частоты возбуждения $(2.97 \mathrm{GHz})$ от частоты собственных колебаний упругой системы $(2.80 \mathrm{GHz})$, т.е. сильные магнитные колебания заставляют упругую систему колебаться на несвойственной ей частоте, в результате чего она оказывает определенное сопротивление. При этом амплитуда как магнитных, так и упругих колебаний имеет сильно изрезанный характер, обусловленный периодическими биениями. Период биений составляет около $5.7 \cdot 10^{-9} \mathrm{~s}$, что соответствует частоте $0.17 \mathrm{GHz}$, т. е. разности между частотой возбуждения и частотой основного резонанса, как это обычно характерно для вынужденных колебаний.

\section{Заключительные замечания}

Основным результатом настоящей работы можно считать демонстрацию расщепления упругого резонанса в случае сильно нелинейного возбуждения магнитной системы, проявляющегося в виде появления двух дополнительных резонансов на отдельных частотах. Выявлен пороговый характер такого расщепления. Установлено, что амплитуда максимального дополнительного резонанса сравнима с амплитудой основного, а частота определяется верхней частотой нелинейной частотной характеристики магнитной системы. Показана возможность управления частотой дополнительного упругого резонанса путем изменения уровня возбуждающего магнитного поля. Отмечено прикладное значение такого управления, не требующего изменения механических параметров системы.

\section{Финансирование работы}

Работа выполнена в рамках государственного задания, а также частично поддержана грантами Правительства 
Республики Коми и РФФИ № 20-42-110004, р_а и грантом РНФ № 21-72-20048.

\section{Конфликт интересов}

Авторы заявляют, что у них нет конфликта интересов.

\section{Список литературы}

[1] И.П. Голямина. В кн. Ультразвук. Маленькая энциклопедия (Советская энциклопедия, М., 1979), с. 196-200.

[2] И.П. Голямина. В сб.: Физика и техника мощного ультразвука. Кн. 1. Источники мощного ультразвука, под ред. И.П. Голяминой (Наука, М., 1967)

[3] R.L. Comstock, R.C. Le Craw. J. Appl. Phys., 34 (10), 3022 (1963).

[4] Р. Ле-Кроу, Р. Комсток. В кн.: Физическая акустика. Т.ЗБ. Динамика решетки, под ред. У. Мэзона (Мир, М., 1968), c. 156. [Пер. с англ. R.C. Le Craw, R.L. Comstock. In book: Physical Acoustics. V. 3. Part.B. Lattice dynamics. (Academic Press, NY. and London, 1965), p. 156.]

[5] E. Schlömann, R.I. Joseph. J. Appl. Phys., 35 (8), 2382 (1964).

[6] С.Н. Полулях, В.Н. Бержанский, Е.Ю. Семук, В.И. Белотелов, П.М. Ветошко, В.В. Попов, А.Н. Шапошников, А.И. Чернов. ЖТФ, 21 (7), 1124 (2021). DOI: 10.21883/JTF.2022.01.51853.167-21-20 [S.N. Polulyakh, V.N.Bershansky, E.Yu. Semuk, V.I. Belotelov, P.M. Vetoshko, V.V. Popov, A.N. Shaposhnikov, A.I. Chernov. Journ. Tech. Phys., 21(7), 1124 (2021).]

[7] А.Г. Гуревич, Г.А. Мелков. Магнитные колебания и волны (Физматлит, М., 1994) [A. Gurevich, G. Melkov. Magnetic Oscillations and Waves (Nauka-Fizmatlit, M., 1994)]

[8] Я.А. Моносов. Нелинейный ферромагнитный резонанс (Наука, М., 1971)

[9] В.С. Львов. Нелинейные спиновые волны (Наука, М., 1987)

[10] Ю.В. Гуляев, П.Е. Зильберман, А.Г. Темирязев, М.П. Тихомирова. ФТТ, 42 (8), 1062 (2000).

[11] Д.И. Семенцов, А.М. Шутый. УФН, 177 (8), 831 (2007).

[12] Th. Gerrits, M.I.Schneider, A.B. Kos, T.J. Silva. Phys. Rev. B, $73(9), 094454(7)$ (2006).

[13] В.С. Власов, Л.Н. Котов, В.Г. Шавров, В.И. Щеглов. Радиотехника и электроника, 54 (7), 863 (2009).

[14] В.С. Власов, В.Г. Шавров, В.И. Щеглов. Журнал радиоэлектроники, 2 (2013). [Электронный ресурс] Режим доступа: http://jre.cplire.ru/jre/feb13/10/text.pdf

[15] В.С. Власов, П.А. Макаров, В.Г. Шавров, В.И. Щеглов. Журнал радиоэлектроники, 6 (2017). [Электронный ресурс] Режим доступа: http://jre.cplire.ru/jre/jun17/5/text.pdf

[16] Ф.Ф. Асадуллин, С.М. Полещиков, Д.А. Плешев, Л.Н. Котов, В.В. Власов, В.Г. Шавров, В.И. Щеглов. Журн. Сибирского федерального ун-та. Серия: Математика и физика, 10 (1), 36 (2017). [F.F. Asadullin, S.M. Poleshchikov, D.A. Pleshev, L.N. Kotov, V.V. Vlasov, V.G. Shavrov, V.I. Shcheglov. J. Siberian University. Mathematics and Physics, 10 (1), 36, (2017).] 